\title{
EPILEPSIA PARCIAL EN NIÑOS
}

\author{
PATRICIA CAMPOS O.* - GENARO HERRERA G.**
}

\begin{abstract}
RESUMEN - Se presenta una serie retrospectiva y prospectiva de 101 paclentes con diagnóstico de epilepsia parcial evaluados en el Consultorio Externo de Neuropediatria del Hospital General Base Cayetano Heredia entre Enero de 1984 y Julio de 1988 con un seguimiento entre 6 meses y 4 años. Las edades oscilaron entre 6 meses y 13 años. Encontráronse 19\% de epilepsias idiopáticas con un $7.7 \%$ del total para epilepsias benignas de la infancia. Del $39 \%$ de epflepsias sintomáticas el mayor porcentaie correspondí́ en orden de frecuencia a secuelas de problemas perinatales, granulomas tuberculosos y secuelas de infecciones en el sistema nervioso central.
\end{abstract}

\section{Partial epilepsy in childhood.}

SUMMARY - It is reported the analysis of a retrospective and prospective study on a series of 101 patients suffering from partial epilepsy who have been examined at the Neuropediatric Clinics of Hospital General Base Cayetano Heredia during the period that lasted from January 1984 to July 1988. The observation time varied from 6 months to 4 years. The patients age varied from 6 months to 3 years old. There were found 19\% of idiopathis epilepsy with $7.7 \%$ from benign epilepsies in childhood. The symptomatic epilepsles corresponded to sequels of problems short after birth, followed by tuberculosis granulomata and sequels of infections in the central nervous system.

Las crisis parciales, por definición, son aquellas que clínica y electroencefalográficamente evidencian compromiso anatómico o funcional de una parte de un hemisferio cerebral. De esta manera la localización del foco EEG determina la forma clinica de la crisis 10,11,23. Para Dam 4 y Delgado-Escueta 5 representan la forma más común de crisis en el adulto $(67 \%)$ y en el niño $(40 \%)$, la mitade de las cuales evolucionan a generalización secundaria. En las crisis parciales se reconocen básicamente dos tipos: parciales simples y parciales complejas, a menudo asociadas a patología orgánica. En la última década se ha venido dando énfasis a un sindrome especial en la infancia con crisis parciales, las llamadas epilepsias benignas de la infancia. Con un cuadro bien definido y usualmente un curso benigno, la más frecuente de ellas, la epilepsia benigna a puntas rolándicas, fue descrita en 1958, por primera vez por Nayrac y Beaussart 21. Menos frecuentes son los reportes acerca de etiología, epidemiología y seguimiento de crisis parciales en niños. En 1982, Scarpa y Carassini 24 , a propósito de 261 casos, revisan las características clínico-EEG con seguimiento a largo plazo. En 1986 Deonna y col.6, en un estudio de 107 niños con exámen neurológico normal, encuentran aproximadamente un tercio de epilepsias benignas de la infancia. Mas recientemente, Watanabe y col.25 estudian retrospectivamente 53 pacientes con epilepsia del lóbulo temporal, encontrando $10 \%$ de etiología tumoral.

El presente estudio tiene por objeto presentar nuestra experiencia a propósito de epilepsias parciales en niños, analizar las características clinico-EEG y hacer una aproximación etiológica, buscando frecuencia de epilepsias benignas.

Departamentos de Neurología * y de Radiología** de la Universidad Peruana Cayetano Heredia, Lima. 


\section{MATERIAL $\mathbf{Y}$ METODOS}

Se revisaron 101 historias clinicas provenientes del Consultorio Externo de Neuropediatria del Hospital General Base Cayetano Heredia, que hubieran consultado como casos nuevas o continuadores entre Enero de 1984 y Julio de 1988, pertenecientes a niños entre 6 meses y 13 años de edad, cuyo motivo de consulta hubiera sido sindrome convulstvo parcial, excluyendo las convulsiones febriles, los cuadros en vigencia de procesos infecciosos $y / 0$ metabolicios agudos $y$ aquellos con datos insuficientes. De esta manera todos los pacientes han sido seguidos por un periodo compreendido entre 6 meses y 4 años.

Los datos obtenidos en todos los casos incluyen: caracteristicas de las crisis, edad de aparición de la primera crisis, frecuencia $y$ horario de las mismas, antecedentes familiares $y$ versonales de epilepsia $y$ convulsión febril, terapia, control de crisis, efectos colaterales de medicación $y$ evaluación de niveles de drogas.

Se tomaron inicialmente 82 EEG $y$ la etlología se precisó con diferentes estudios: TAC en 39 pacientes, gammagrafia cerebral en 9 , arteriografia carotıdea en 3 , radiografia de cráneo en 48 y estudio de líquido céfaloraquídeo en 26.

\section{RESULTADOS}

Con respecto a edad y sexo fueron 58\% de escolares, 37\% de pre-escolares y únicamente $6 \%$ de lactantes, no habiendo predominio de ningún sexo (52\% femenino y $48 \%$ masculino). En relación a antecedentes, $32 \%$ tenían historia de cuadros convulsivos febriles y no febriles en sus famillares cercanos, $26 \%$ habian tenido cuadros convulsivos o convulsión febril previa, 17\% hístoria de TEC sin mayores factores de riesgo, 12\% historia de problemas perinatales y atraso en el desarrollo psicomotor, y $9 \%$ antecedente de proceso infeccioso en el sistema nervioso central (SNC).

Las crisis fueron en 91\% (92 pecientes) parciales simples y en 9\% parciales complejas; de las parciales simples las más frecuentes fueron las motoras (57\%) seguidas de las versivas $(17 \%)$; hubieron 6 crisis visuales, 2 f(natorias y 4 con sintomatologia psiquica; y entre las parciales complejas prácticamente el mismo número con $y$ sin automatismos. Unicamente 39 (39\%) permanecieron siendo parciales puras, la gran mayoría tuvieron generalización secundaria (Tabla 1). La edad de aparición de las crisis varió entre 1 mes y 13 años, encontrándose el 50\% después de los 6 años y únicamente $10 \%$ en lactantes antes de los 2 años. En relación a frecuencia: fue crisis única en $37 \%$, rara (no mas de 1 cada 6 meses) en $23 \%$, frecuente (con intervalo de 1 a 6 meses) en $14 \%$ y muy frecuente (más de 1 as mes) en 24\%; en el 2\% se desconoce la frecuencia. En 57 casos (56\%) no se encontró factor desencadenante y del $\mathbf{2 4 \%}$ que tuvo este factor, el más comúnmente encontrado fue la supresión brusca de tratamiento $(50 \%)$.

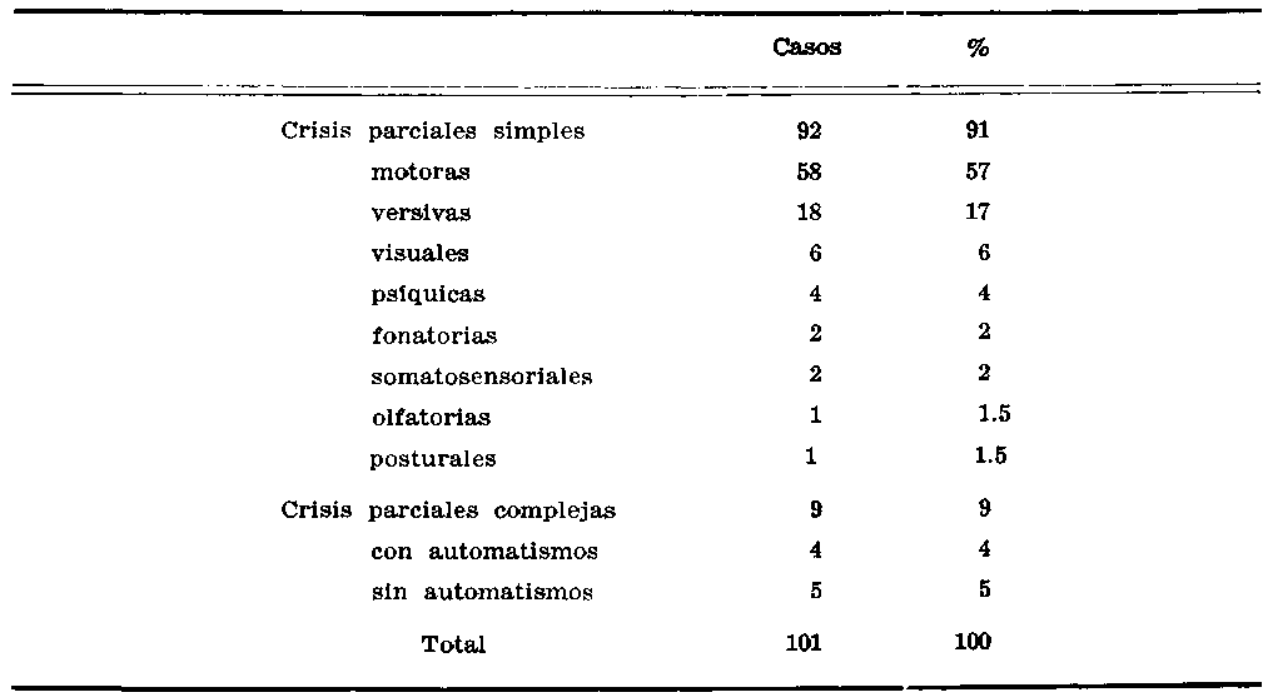


5l exámen neurológico fue normal en 63 pacientes (64\%) y de los hallazgos anormales el más frecuente fue retardo mental (13 casos) seguido de cuadros de déficit motor (9 casos); únicamente en 3 casos hubieron sintomas $y$ signos de hipertensión endocraneana. Con respecto a exámenes auxiltares: se realizó estudio de liquido céfaloraquideo en 26 pactentes encontrandose anormal en 10, se obtuvieron 48 radiografias de craneo las que fuerom anormales en 13 (4 correspondieron a calcificaciones, 3 a signos de hipertensión endocraneana entre otras). Se realizó TAC en 39 pacientes siendo el 50\% anormales (Fig. 1), de las 3 arteriografías sólo fue anormal la de la MAV.

De los 82 EAgG inicialmente realizađos, 68 (83\%) fueron anormales, de éstos $60 \%$ con actividad paroxistica siendo más del 50\% actividad paroxística focal: 10 focos temporales, 3 rolándicos, 2 centro-parietales, 2 fronto-temporales, 1 trontal y 1 occipital. El resto se repartió más o menos de la siguiente forma: actividad paroxística difusa en 13 pacientes, multifocal en 2 y anormalidades de actividad de base en 22 pacientes siendo tocn de ondas lentas en 9, no siempre coincidiendo con lesión estructural (2 de 9 tuvieron TAC normal).

Con respecto a etiologia: 19\% (19 pacientes) fueron idiopaticas, 39\% (39 pacientes) sintomáticas $y$ en $43 \%$ (43 pacientes) no se pudo determinar la misma por no haberse podido completar los estudios. De las epilepsias sintomáticas o secundarias, la causa más frecuente fue la secuela de problemas perinatales (28\%), seguida de granulomas probablemente tuberculosos (17\%) y de secuelas de infecciones en el SNC (15\%). Hubo también un 15\% de pacientes en los que no se pudo definir la imágen tomografica; de ellos, 6 fueron informados como probable cisticercosis, la misma que se pudo comprobar en 3 . Hubleron 3 casos de malformaciones congénitas (hidrocefalia $v$ quiste porencefálico) una de ellas asociada $a$ un sindrome de Pierre-Robin, dos pacientes con epilepsia post-traumática y un caso de cada una de las siguientes etiologias: malformación A-V, atrofia córtico-subcortical, edema frontal y hematoma subdural crónico laminar (Tabla 2).

\section{Casos \%}

Secuela de problemas perinatales

Granulomas TBC

Secuela de infecciones SNC

Imágenes $\mathrm{TAC}$ no concluyentes

Malformaciones congénitas SNC

Post traumática

Malformación A-V

Atrofia córtico-subcortical

Edema frontal

Hematoma subdural laminar

Total

$\begin{array}{rr}11 & 28 \\ 7 & 17 \\ 6 & 15 \\ 6 & 15 \\ 3 & 8 \\ 2 & 5 \\ 1 & 3 \\ 1 & 3 \\ 1 & 3 \\ 1 & 3 \\ 39 & 100\end{array}$

Tabla 2-Epilepsias secundarias.

En el 72\% se usó monoterapia y únicamente un paclente necesitó 3 drogas en su tra, tamiento. En orden de frecuencia las drogas más usadas fueron: difenilhidantoina, fenobarbital, carbamazepina y primidona; habiéndose encontrado efectos colaterales en 11\% de los pacientes, la mayor parte de ellos en relación a la difenilhidantoina y la mayuría con sintomatología neurológica (ataxia, temblor, somnolencia).

En el seguimiento a 24 meses $72 \%$ estaban controlados total o parcialmente, 9\% sin control $y$ en $19 \%$ no se pudo establecer la situación clínica. En el seguimiento a 48 meses $87 \%$ estaban total o parcialmente controlados. El seguimiento EEG fue más dificil de realizar bdisicamente por motivos económicos, sin embargo es interesante señalar que a los 24 meses de 20 EEG normales el número subió a 37 y se encontró actívidad paroxistica focal únicamente en 2. 


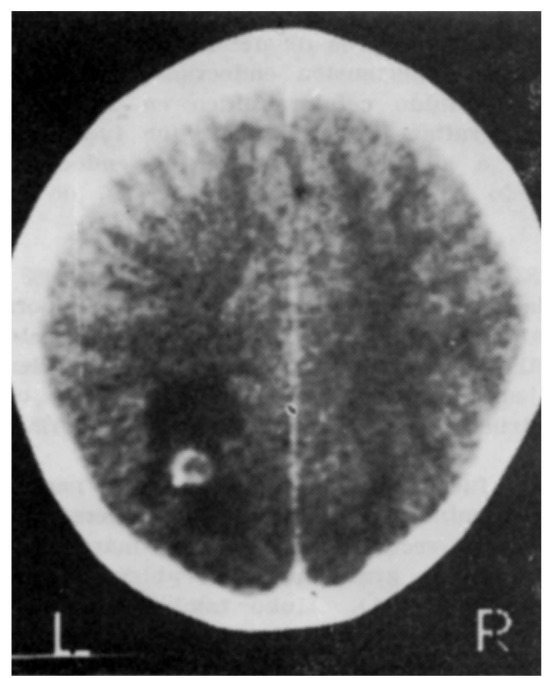

paciente con hemiplejia congénita y crisis motoras en hemicuerpo derecho.

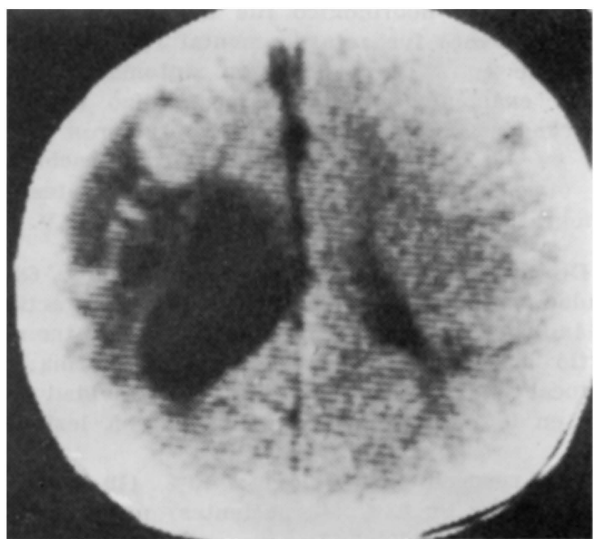

Fig. 1 - Izquierda: tuberculoma cerebral; paciente con crisis parcial motora en miembro superior derecho $y$ hemiparesia sin afasia. Derecha: quiste porencefalico; T'ARIOS

En relación a etiologia de las crisis parciales, desde que en 1970 Gastaut las asociara a una gran variedade de lesiones locales, se han descrito muchos cuadros causantes de ellas, siendo la secuela de problemas perinatales la causa más común. Al respecto, O'Donohoe 22 enfatiza que el dato de un parto no complicado no excluye el hecho de que haya habido una pequeña lesión isquémica temporal causante de epilepsia secundaria. En orden de frecuencia las lesiones por secuela de procesos infecciosos ocupan el segundo lugar seguidas por las secuelas de TEC. Raramente en la infancia se sospechan procesos expansivos, pero el mismo advenimiento de métodos de auxilio diagnóstico cada vez más sofisticados hace que el procéso expansivo en sentido amplio sea una prioridad, y en paises en desarrollo los procesos infecciosos-inflamatorios deben tomarse en mayor consideración. Hosking 15 llamó la atención para las derivaciones ventrículo-peritoneales como causa de crisis parciales. Harbord y Manson 13 encontraron que el factor etiológico más frecuente, en su casuística de 53 niños, era el antecedente de crisis febril complicada; en segundo lugar los procesos expansivos neoplásicos (4 astrocitomas entre otros) y en menos del $8 \%$ sccuelas de problemas perinatales y encefalitis. Scarpa y Carassini 24 encontraron $29 \%$ de secuelas de problemas perinatales, $21 \%$ con historia familiar positiva, $10 \%$ de secuelas post TC y únicamente $4.5 \%$ de secuelas de infecciones.

En nuestra serie, indistintamente entre los portadores de epilepsia idiopática y secundaria, hubo un $32 \%$ de historia familiar de epilepsia o de convulsión febril. En $26 \%$ de los pacientes habia historia previa de sindrome convulsivo o convulsión febril, la mayoría de ellas benignas. Del porcentaje total de epilepsias secundarias, en orden de frecuencia la mayoria correspondió a secuelas de problemas perinatales y ese fue el grupo con mayor incidencia de exámen neurológico anormal. En segundo lugar tuvimos los procesos inflamatorios catalogados como tuberculomas porque reunieron por to menos tres de los siguientes criterios: PPD mayor de $10 \mathrm{~mm}$, historia de contacto TBC, radiografía de tórax anormal, aislamiento de BK y prueba terapeútica; ninguno de ellos tuvo comprobación quirúrgica. El tercer lugar correspondio a las stcuelas de procesos infecciosos bacterianos o virales. No hubo cualquier proceso neoplásico. En relación a TEC sólo en dos casos fue posible demonstrar que la epilepsia era post traumática, en el resto con este antecedente o se demonstró otra etiologia o el TEC no reunia las características para ser considerado factor de riesgo.

En relación a tratamiento y evolución clínica, es evidente que en cualquier edad de la vida la monoterapia es un axioma de terapia en epilepsia, desde que cada vez más se aplica la farmacodinamia para un mejor control. Al respecto quisiéramos 
resaltar el control en $72 \%$ a los 24 meses de seguimiento considerando el casi $20 \%$ de epilepsias secundarias.

Con respecto a las llamadas «epilepsias benignas de la infancia» 2,7 , desde que en 1958 Nayrac y Beaussart 21 describieron por primera vez la epilepsia rolándica, el cuadro se ha ido complementando con los aportes sucesivos de Faure y Loiseau (1960), Lombroso $(1967)^{19}$, Lerman y Kiviky $(1975)^{20}$, Fejerman y DiBlasi $(1987)^{9}$ y Loiseau y col. (1988)18. En años recientes han sido identificadas otras formas de epilepsia benigna, con foco temporal (Dalla Bernardina y col.3, Watanabe y col.25), parietal (DeMarco 7) y occipital (Gastaut 12, Kuzniecky y Rosenblatt 16). En nuestra casuística llama la atención que únicamente 8 casos (7.7\% del total) tienen características clínicas de epilepsias benignas, 7 con sintomatología rolándica y uno con sintomatologia de epilepsia con pautas occipitales. De los 7 primeros únicamente 3 tenían EEG anormal; actualmente todos ellos tienen un seguimiento mayor de 3 años, están completamente asintomáticos e inclusive uno sin terapia. Otro de los pacientes presentó un EEG con complejos punta-onda 3.5-5 pero sin evidencia clínica de ausencias concomitantes tal como fuera descrito por Aicardi y Chevrie 1 . La paciente con sintomatologia afectiva no tuvo EEG con puntas occipitales y el foco occipital correspondió a una crisis sensori-motora.

Conclusiones - 1. En nuestra casuística la mayor frecuencia de crisis correspondió a las parciales simples con sintomatología de tipo elemental. 2. Un tercio de los pacientes tuvieron antecedente familiar de epilepsia o convulsión febril, y un tercio también historia previa de sindrome convulsivo, la mitad de los cuales fue convulsión febril no complicada. 3. En relación a etiología el $19 \%$ fue idiopática, correspondiendo las epilepsias benignas a un $7.7 \%$ del total. 4. En el grupo de epilepsias secundarias $(39 \%)$, la causa más frecuente fue la secuela de problemas perinatales seguida de procesos inflamatorio-infecciosos (TBC) y de secuelas de infecciones en el SNC. 5. En el seguimiento a los 24 meses $72 \%$ estaban parcial o totalmente controlados y habia un $30 \%$ aproximadamente de normalización del EEG.

\section{REFERENCIAS}

1. Aicardi J, Chevrie $J$ - A typical benign epilepsy of childhood. Dev Med Child Neurol $24: 281,1982$.

2. Beaussart $M-$ Benign epilepsy of children with rolandic (centro temporal) paroxysmal focl: a clinical entity. Study of 221 cases. Epilepsia 13:795, 1972.

3. Dalla Bernardina B, Bureau M, Dravet C, Dulac C, Tassinari CA, Roges J - Epilépsie bénigne de l'enfant avec crises a semiologie affective. Rev Neurophysiol 10:8, 1980.

4. Dam M, Kiorbe E - Epilepsy diagnosis and treatment. Scriptor, Copenhagen, 1980.

5. Delgado-Escueta A $\rightarrow$ Summation of Kroc Foundation workshop and discussion: the new wave of research in the epilepsies. Ann Neurol 16:140, 1984.

6. Deonna T, Ziegler AL, Despland PA, Van Melle G - Partial epilepsy in neurologically normal children: clinical syndromes and prognosis. Epilepsia 27:241, 1986.

7. DeMarco P, Tassinari CA - Extreme somatosensory evoked potentials: an EEG sign forecasting the possible occurrence of seizures in children. Epilepsia $22: 569,1981$.

8. Feierman N, Medina CS -- Convulsiones en la Infancia: Diagnóstico y Tratamiento. Ed 2. Ateneo, Buenos Aires, 1986.

y. Fejerman N, DiBlasi M - Status epllepticus of benign partial epilepsies in children: report of two cases. Epllepsia $28: 351,1987$.

10. Gastaut $\mathbf{H}-$ Clinical and electroencephalographic classification of epileptic seizures. Epllepsia 11:102, 1970.

11. Gastaut H, Gastaut L, Gonçalves e Silva GE, Fernandez Sanchez GR -- Relative frequency of different types of epilepsy: a study employing the classification of the I.L.A.E. Epilepsia 16:457, 1977.

12. Gastaut HI - A new type of epilepsy: benign partial epilepsy of childhood with occipita] spike-waves. Clin EEG 13:13, 1982. 
13. Harbord MG, Manson JI - Temporal lobe epilepsy in childhood: reappraisal of etiology and outcome. Pediatr Neurol $3: 263,1987$.

14. Heijbel J, Blom S, Rasmussen $M-$ Benign epilepsy of childhood with centrotemporal EEG foci: a genetic study. Epilepsia 16:285, 1975.

15. Hosking GP - Fits in hydrocephalic children. Arch Dis Child 49:033, 1974.

16. Kuzniecky R, Rosenblatt $B$ - Benign occipital epilepsy: a family study. Epilepsia 28:346, 1987.

17. Loiseau $P$, Beaussart $M$ - The seizures of benign childhood epilepsy with rolandic paroxysmal discharges. Epilepsla 14:381, 1973.

18. Loiseau P, Duché B, Córdova $\mathbf{S}$, Dartigues JF, Cohsdon $\mathbf{S}-$ Prognosis of benign childhood epilepsy with centrotemporal spikes: a follow-up study of 168 patients. Epilepsia 29:229, 1988.

19. Lombroso C - Sylvian seizures and midtemporal spike foci in children. Arch Neurol $17: 52,1967$.

20. Lerman $P$, Kivity $S \longrightarrow$ Benign focal epilepsy of childhood: a follow-up study of 100 recovered patients. Arch Neurol $32: 261,1975$.

21. Nayrac $\mathbf{P}$, Beaussart $\mathbf{M} \rightarrow$ Les pointes-ondes pré-rolandiques: expression EFG très perticulière: étude électrnclinique de 21 cas. Rev Neurol 99:201, 1958.

22. O'Donohoe N - Epilepsies of Childhood. John Apley, London, 1979.

23. Rasmussen $\mathrm{T}$ - Selzures with local onset and elementary symptomatology. In Vinken PJ, Bruyn GW (eds): Handbook of Clinical Neurology, Vol 15. North Holland, Amsterdam, 1974.

24. Scarpa P, Carassini B - Partial epilepsy in childhood: clinical and EEG study of 261 cases. Epllepsia 23:333, 1982.

25. Watanabe K, Yamamoto N, Negoto T, Takaesu E, Aso K, Furune S, Takahashi I Benfon complex partial epilepsies in infancy. Pediatr Neurol 3:208, 1987. 